\title{
Characterization of long time fluctuations of forces exerted on an oscillating circular cylinder at $\mathrm{KC}=10$
}

\author{
Marion Duclercq ${ }^{\mathrm{a}, \mathrm{b}, *}$, Daniel Broc ${ }^{\mathrm{a}}$, Olivier Cadot $^{\mathrm{b}}$ \\ a CEA, DEN, DM2S, SEMT, EMSI, F-91191 Gif-sur-Yvette, France \\ ${ }^{\mathrm{b}}$ ENSTA-ParisTech, Chemin de la Hunière, 91761 Palaiseau, France
}

Flow dynamics, in-line and transverse forces exerted on an oscillating circular cylinder in a fluid initially at rest are studied by numerical resolution of the two-dimensional Navier-Stokes equations. The Keulegan-Carpenter number is held constant at $\mathrm{KC}=10$ and $\mathrm{Re}$ is increased from 40 to 500 . For the different flow regimes, links between flow spatio-temporal symmetries and force histories are established. Besides simulations of long duration show that in two ranges of Re, forces exhibit low frequency fluctuations compared to the cylinder oscillation frequency. Such observations have been only mentioned in the literature and are more deeply examined here. In both ranges, force fluctuations correspond to oscillations of the front and rear stagnation points on the cylinder surface. However, they occur in flow regimes whose basic patterns ( $V$-shaped mode or diagonal mode) have different symmetry features, inducing two distinct behaviors. For $80 \leq \operatorname{Re} \leq 100$, fluctuations are related to a spectral broadening of the harmonics and to a permutation between three vortex patterns ( $\mathrm{V}$-shaped, transverse and oblique modes). In the second range $150 \leq \operatorname{Re} \leq 280$, amplitude fluctuations are correlated to the appearance of low frequency peaks interacting with harmonics of the cylinder frequency. Fluctuations are then a combination of a wavy fluctuation and an amplitude modulation. The carrier frequency corresponding to the wavy fluctuation depends on Re and is related to a fluid characteristic time; the modulation frequency is independent of Re and equal to $1 / 4$ of the cylinder oscillation frequency.

\author{
Keywords: \\ Oscillating circular cylinder \\ Keulegan-Carpenter number \\ Finite element method \\ In-line force \\ Transverse force \\ Vortex dynamics
}

\section{Introduction}

The case of a cylinder oscillating in a fluid otherwise at rest is of fundamental importance since it provides a simplified model of wave-structure interactions in offshore (e.g. riser tubes and pipes subjected to currents in deep water) or nuclear engineering (e.g. stream generators, heat exchangers or fuel assemblies subjected to seismic excitations in reactor cores with pressurized water or sodium). This paper presents results of a two-dimensional numerical investigation of a rigid circular cylinder undergoing rectilinear sinusoidal oscillations transversely to its long axis in a quiescent viscous fluid. The system can be described by two dimensionless numbers, the Keulegan-Carpenter number, $K C=U_{0} T / D$, and the Reynolds number, $\operatorname{Re}=U_{0} D / v$, where $U_{0}$ is the maximum cylinder velocity, $T$ the cylinder oscillation period, $D$ the cylinder diameter

\footnotetext{
* Corresponding author at: CEA, DEN, DM2S, SEMT, EMSI, F-91191 Gif-sur-Yvette, France. Fax: +3316908 8331.

E-mail address: marion.duclercq@ensta.org (M. Duclercq).
} 
and $v$ the kinematic viscosity of the fluid. The ratio $\beta=\operatorname{Re} / \mathrm{KC}=D^{2} /(v T)$, named frequency parameter or Stokes number (see Sarpkaya, 2005), can also be used instead of Re.

Most of the analyses regarding the in-line force $\mathcal{F}_{x}$ exerted by the fluid on the cylinder relate to the determination of the inertia and drag coefficients $C_{m}$ and $C_{d}$ defined by the decomposition of Morison et al. (1950).

$$
\mathcal{F}_{x}^{\text {Morison }}(t)=C_{m} \frac{\rho \pi D^{2}}{4} \frac{d U_{\text {cyl }}}{d t}(t)+C_{d} \frac{1}{2} \rho D\left|U_{\text {cyl }}(t)\right| U_{\text {cyl }}(t)
$$

where $\rho$ is the fluid density, $U_{\text {cyl }}(t)=U_{0} \sin (2 \pi t / T)$ the $x$-component of the cylinder velocity, and $C_{m}$ and $C_{d}$ must be determined empirically, for example from a Fourier series decomposition. However, at sufficiently low KC, such that the flow remains laminar, attached and two-dimensional, it is possible to obtain analytical solutions. A theoretical analysis by Wang (1968) showed that under those hypotheses, $C_{m}$ and $C_{d}$ can be expressed in terms of KC and $\beta$. The classical approach to describe the force in the general case consists in determining $C_{m}$ and $C_{d}$ versus KC and $\beta$ or Re (e.g. Obasaju et al., 1988; Justesen, 1991; Smith and Stansby, 1991; Lin et al., 1996; Sun and Dalton, 1996; Zhang and Zhang, 1997; Dütsch et al., 1998; Iliadis and Anagnostopoulos, 1998; Uzunoğlu et al., 2001; Sarpkaya, 2005). However, significant discrepancies appear for higher KC. Eq. (1) is not efficient anymore as soon as vortex shedding occurs, since force signals are not sinusoidal anymore. Then researches also directed toward flow analysis.

From experiments for $0<\mathrm{KC}<32$ at $\beta=730$ or 255, Williamson (1985) defined different flow regimes whose boundaries only depend on $\mathrm{KC}$. For $\mathrm{KC}<7$, pairing of attached vortices occurs, symmetrical then asymmetrical. For $6<\mathrm{KC}<13$ a one-sided transverse street appears with a jet of vortices moving away roughly perpendicular to the oscillation direction. For $13<\mathrm{KC}<15$ (single-pair regime), a one-sided street is still observable but it is inclined at $45^{\circ}$. The range $15<\mathrm{KC}<24$ is referred to as double pair regime, since two pairs of vortices are formed in each cycle and convected away in opposite directions and from opposite quadrants. From KC=24 flows consist of three pairs, then four pairs of vortices. Later, through experimental or numerical studies, Obasaju et al. (1988), Justesen (1991), Lin et al. (1996), Lam and Dai (2002) and Lam et al. (2010) confirmed the results of Williamson (1985) for high $\beta$ and identified mechanisms governing the development of vortices and their interactions (Lam et al., 2010).

Meanwhile, Tatsuno and Bearman (1990) visualized flow structure for $5<\beta<160$ and $1.6<$ KC $<15$. Three-dimensional features were also examined. Eight regimes A to $G$ were identified. Their boundaries were drawn in the plane $(\beta, \mathrm{KC})$. This figure is reproduced here in Fig. 1 , but in the plane $(\mathrm{KC}, \mathrm{Re})$. Regimes $\mathrm{A}^{*}$ and $\mathrm{A}$ correspond to the symmetrical regime of Williamson (1985). Regime B is similar but presents a three-dimensional structure in the axial direction. Regime $\mathrm{C}$ is comparable to a von Kármán vortex street but with vortices of opposite senses of rotation. Regime D presents a $\mathrm{V}$-pattern symmetrical about the transverse axis. Flow in regime $\mathrm{E}$ is identical but the V-pattern intermittently changes its direction from one side to the other. Regime F corresponds to the double pair regime of Williamson (1985). Regime G is similar to the transverse street observed by Williamson (1985). Thus the investigations of Tatsuno and Bearman (1990), focused on lower $\beta$ than Williamson (1985), revealed that flow regimes cannot be defined anymore from KC only, because $\operatorname{Re}($ or $\beta$ ) becomes also decisive if smaller. The present paper shall highlight the importance of Re since we consider a fixed value of $\mathrm{KC}=10$ and study the influence of increasing Re up to 500. Many authors such as Iliadis and Anagnostopoulos (1998), Dütsch et al. (1998), Uzunoğlu et al. (2001), Guilmineau and Queutey (2002) or Nehari et al. (2004) selected a few points in the map established by Tatsuno and Bearman (1990) to detail descriptions of the corresponding regimes. They obtained a good agreement with the main results of Tatsuno and Bearman (1990).

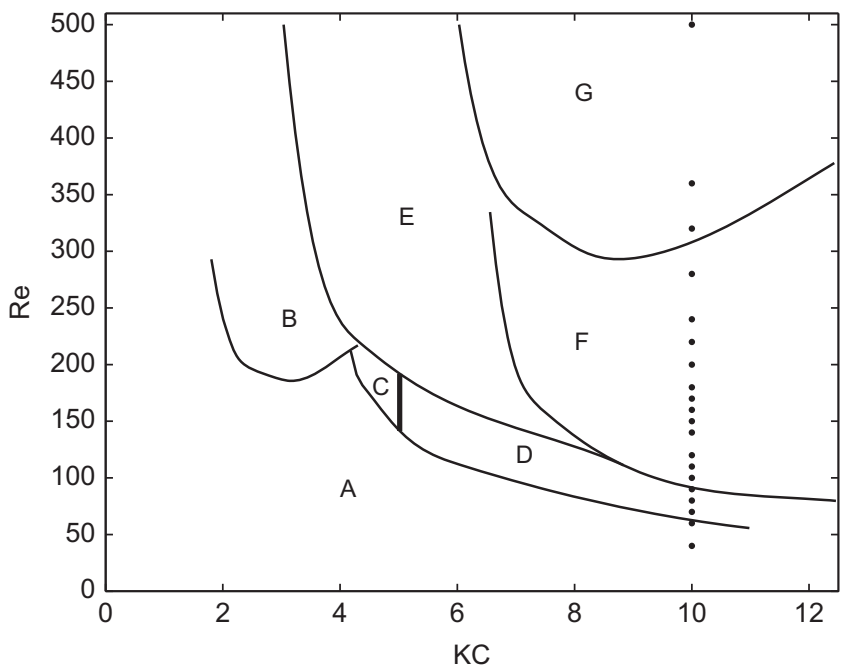

Fig. 1. Flow regimes A to G (see text) defined by Tatsuno and Bearman (1990). Dots show the numerical simulations of the present study. 
However, if every author reported identical descriptions of the flow patterns, some discrepancies appear regarding their stability, for instance in regime D. More recently, Elston et al. $(2004,2006)$ effected two- and three-dimensional Floquet analyses to study symmetry breaking transitions for $\mathrm{KC}<10$ and $\beta<100$. From symmetrical regime A they obtained a single curve of two-dimensional marginal stability, but bifurcations on it fell into two distinct regimes. The first transition for low $\beta$ and high KC yields synchronous modes, which is actually regime $\mathrm{D}$. The second one for higher $\beta$ leads to quasiperiodic modes since a secondary period arises. This corresponds to the transition from regimes A to $C$. In both cases, flows in regimes $C$ and $D$ rapidly saturate then are unstable to three-dimensional secondary instabilities. At $\beta>50$, the primary instability is three-dimensional, producing regime B. Besides, using two- and three-dimensional models, Nehari et al. (2004) showed that two-dimensional features of the flows in regimes D and F were not qualitatively affected by threedimensional effects. Hence, it is meaningful to perform two-dimensional computations in that range of (KC, Re).

The objective of this paper is to provide a better description and understanding of the system behavior for low Re values. The analysis is carried out for long durations compared to the cylinder oscillation period. Indeed some flow patterns seem aperiodic, yielding force fluctuations as indicated by Dütsch et al. (1998), Uzunoğlu et al. (2001) and Elston et al. (2006). We study here those fluctuations and their links with flow dynamics. In complement to most of the previous researches focused on the influence of $\mathrm{KC}$, we concentrate here on the role of Re. Numerical simulations are carried out at $\mathrm{KC}=10$ and Re values from 40 to 500 . The $\mathrm{KC}$ value is chosen as 10 because it enables encountering a great number of regimes (A, D, F and G according to Fig. 1) whose flow structure is two-dimensional. In what follows, Section 2 gives precisions about numerical method. Then Section 3 presents forces and flows when Re increases. Successive ranges of Re appear corresponding to different behaviors of the system. Transitions between regimes are characterized, and amplitude fluctuations of the forces are highlighted then discussed in Section 4.

\section{Numerical method}

The mathematical model consists in the two-dimensional incompressible Navier-Stokes equations in the primitive variables formulation:

$$
\left\{\begin{array}{l}
\operatorname{div}(\boldsymbol{u})=0 \\
\frac{\partial \boldsymbol{u}}{\partial t}+\mathrm{KC} \boldsymbol{u} \cdot \nabla \boldsymbol{u}=-\mathrm{KC} \nabla p+\frac{\mathrm{KC}}{\operatorname{Re}} \Delta \boldsymbol{u},
\end{array}\right.
$$

where $\boldsymbol{u}$ and $p$ are the velocity and pressure fields in the fluid nondimensionalized by $U_{0}$ and $0.5 \rho U_{0}^{2}$, respectively. Times are nondimensionalized by the oscillation period $T$ and lengths by the cylinder diameter $D$.

A finite element method is used to solve Eq. (2) for the configuration of a cylinder horizontally oscillating in the center of a rectangular fluid domain (Fig. 2(a)). Absolute velocity is computed for a mesh following the cylinder motion: for any time level, the mesh velocity at every node is the cylinder velocity. Moreover outer boundaries are considered to be far enough from the cylinder, so that the fluid remains still. Thus we obtain an arbitrary Lagrangian-Eulerian formulation without mesh deformation given by

$$
\begin{cases}\operatorname{div}(\boldsymbol{u})=0 & \text { in } \Omega_{f} \\ \frac{\partial \boldsymbol{u}}{\partial t}+\mathrm{KC}\left(\boldsymbol{u}-\boldsymbol{U}_{\text {cyl }}\right) \cdot \nabla \boldsymbol{u}=-\mathrm{KC} \nabla p+\frac{\mathrm{KC}}{\operatorname{Re}} \Delta \boldsymbol{u} & \text { in } \Omega_{f} \\ \boldsymbol{u}=\boldsymbol{U}_{\mathrm{cyl}} & \text { on } \Gamma_{\text {cyl }} \\ \boldsymbol{u}=\mathbf{0} & \text { on } \Gamma_{\text {out }} \\ \boldsymbol{u}=\mathbf{0} & \text { at } t=0 \text { in } \Omega_{f},\end{cases}
$$

where $\boldsymbol{u}$ is now the absolute velocity expressed in the reference frame linked to the cylinder, $\Omega_{f}$ is the fluid domain, $\Gamma_{\text {out }}$ its outer boundary and $\Gamma_{\text {cyl }}$ the cylinder boundary. The cylinder dimensionless velocity is $\boldsymbol{U}_{\text {cyl }}=\sin (2 \pi t / T) \boldsymbol{e}_{x}$ where $\boldsymbol{e}_{x}$ and $\boldsymbol{e}_{y}$ are the unit vectors of the reference frame.

Spatial discretization is effected with Crouzeix-Raviart quadrilateral elements; velocity is quadratic and pressure is linear per element but discontinuous on element boundaries (Vuik and Segal, 2006). Eq. (3) is integrated using a DNS algorithm with a second-order finite-difference temporal scheme for accurate prediction of the flow. The problem is solved

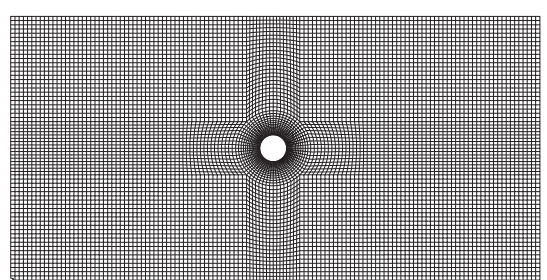

(a)

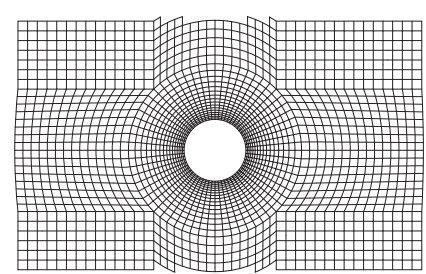

(b)

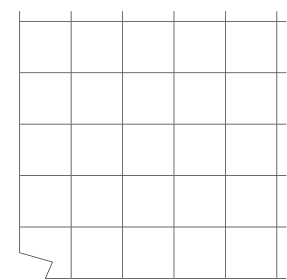

(c)

Fig. 2. (a) Geometry and mesh of the computational domain, zooms on (b) the cylinder and (c) the bottom left-hand corner of the fluid domain. 
by a projection method with internal iterations inside each time level to compute the non-linear convective term with a fixed point method. Then forces on the cylinder are directly deduced from the variational formulation. This method is more accurate than the classical one using pressure and velocity on the cylinder surface.

The mesh consists of squares progressively curved in the vicinity of the cylinder (Fig. 2). The grid refinement around the cylinder (Fig. 2(b)) is guided by the boundary layer thickness around an oscillating flat plate $\delta / D=(4 / \sqrt{2 \pi}) \times(\sqrt{\mathrm{KC} / \mathrm{Re}})$ where $\delta$ is the distance from the plate at which $u / U_{0}$ is $6 \%$ (Iliadis and Anagnostopoulos, 1998). Besides the point at the bottom left-hand corner of the mesh (Fig. 2(c)) is moved of $\boldsymbol{u}_{\text {depl }}$ given by

$$
\frac{1}{D} \boldsymbol{u}_{\text {depl }}=0.1 \boldsymbol{e}_{\boldsymbol{x}}+0.05 \boldsymbol{e}_{\boldsymbol{y}}=d x\left(0.6 \boldsymbol{e}_{\boldsymbol{x}}+0.3 \boldsymbol{e}_{\boldsymbol{y}}\right),
$$

where $d x$ is the dimensionless grid refinement. The local mesh deformation is used to break the symmetry of the geometry and fasten the growing of flow instabilities in the cases of asymmetrical patterns mentioned in the literature. It has also been verified that such a geometric disturbance did not affect symmetrical flow patterns.

A sensitivity analysis of the computational parameters has been carried out to fix the time level $d t$, the grid refinement $d x$, the length $L$ and the height $H$ of the fluid domain, and the number of internal iterations $N_{i}$ inside each time level. Following the same method as Anagnostopoulos and Minear (2004), we investigated the influence of the parameters on the variance of the in-line force coefficient $\left\langle F_{x}(t)^{2}\right\rangle$ where $F_{x}(t)=\mathcal{F}_{x}(t) /\left(\rho D U_{0}^{2}\right)$ is the dimensionless in-line force coefficient and $\langle\cdot\rangle$ the time-averaging operator over the steady state. Finally all the following computations are performed for $d t=5 \times 10^{-3}, d x=0.157, L=20, H=10$ and $N_{i}=4$.

\section{Results}

This section presents the results of simulations effected for $K C=10$ and $40 \leq \operatorname{Re} \leq 500$ (see Fig. 1 ). The transient state at the beginning of each run is not considered, since the investigation deals with steady state. Unlike most of the previous studies where regimes were defined from the flow structure, we propose here to identify regimes from force signals first.

\subsection{Identification of regimes for increasing Re}

\subsubsection{Temporal analysis of the forces}

Different types of time series of forces successively appear as Re increases. For each identified range of Re, an example is provided in Figs. 3 and 4 for the in-line and transverse forces, respectively, with the same axes scales for every sub-figure. For $40 \leq \operatorname{Re} \leq 70$, the in-line force is perfectly periodic and almost sinusoidal (Fig. 3(a)). The transverse force is negligible (Fig. 4(a)). For $80 \leq \operatorname{Re} \leq 100$, the amplitude of the in-line force fluctuates (Fig. 3(b)). The transverse force presents four extrema per period (Fig. 4(f)). Its amplitude also fluctuates and its mean value is not zero anymore (Fig. 4(b)). Fluctuations are regular for $\operatorname{Re}=80$, stronger for $\operatorname{Re}=90$, then irregular for $\operatorname{Re}=100$. For $110 \leq \operatorname{Re} \leq 140$, the in-line force is again perfectly periodic (Fig. 3(c)), but contrary to the range $40 \leq \operatorname{Re} \leq 70$, its amplitude is reduced and curves are sharper (Fig. 3(f)). The transverse force is also periodic (Fig. 4(c)) with six extrema per period (Fig. 4(f)). For $150 \leq$ Re $\leq 280$, the in-line and transverse forces present the same characteristics as previously but their amplitudes fluctuate (Figs. 3(d) and 4(d)). For $320 \leq \operatorname{Re} \leq 500$, forces are chaotic and strong peaks appear (Figs. 3(e) and 4(e)). There are generally 4-7 extrema per cycle for the transverse force.

Fig. 5 shows the variance versus Re of the in-line and transverse forces $\left\langle F_{x}(t)^{2}\right\rangle_{T_{n}}$ and $\left\langle F_{y}(t)^{2}\right\rangle_{T_{n}}$ computed at each $n$th period of the run, and their minimal, mean, and maximal values over the complete run. This representation highlights intrinsic features of the different regimes and transitions between them. The figure also indicates regime boundaries for KC=10 supplied by experiments of Tatsuno and Bearman (1990) between regimes A, D, F and G, and by the stability analysis of Elston et al. (2006) for the transition A-D. Our findings coincide with their critical Re values. On one hand, mean values $\left\langle F_{x}(t)^{2}\right\rangle$ and $\left\langle F_{y}(t)^{2}\right\rangle$ show the global influence of increasing Re on force amplitudes; the in-line force decreases while the transverse force increases. A sudden change appears around $R e=110$. The end of the amplitude fluctuations induces higher values of the in-line force and lower values of the transverse force. For Re $\geq 320$, the transverse force sometimes reaches the same order of magnitude as the in-line force. On the other hand, the display of the points $\left\langle F_{X}(t)^{2}\right\rangle_{T_{n}}$ and $\left\langle F_{y}(t)^{2}\right\rangle_{T_{n}}$ for each simulated period shows if forces are periodic or not. Regimes for $40 \leq$ Re $\leq 70$ and $110 \leq \operatorname{Re} \leq 140$ are periodic because the points are superimposed. On the contrary aperiodic regimes are made visible by the scatter of the points. Those amplitude fluctuations of the force time signals are characterized in the next section from a spectral analysis, which is a natural approach for studying phenomena of long duration.

\subsubsection{Spectral analysis of the forces}

Figs. 6 and 7 present the influence of increasing Re on power spectral densities of the in-line and transverse forces $H_{x(f)}$ and $H_{y(f)}$ defined by

$$
H_{k}(f)=\left|\frac{1}{100 T} \int_{t_{i}}^{t_{i}+100 T} e^{-i 2 \pi f t} F_{k}(t) d t\right|^{2}, \quad k=x, y,
$$

where $\left[t_{i}, t_{i}+100 T\right]$ is a time interval in the steady state. 

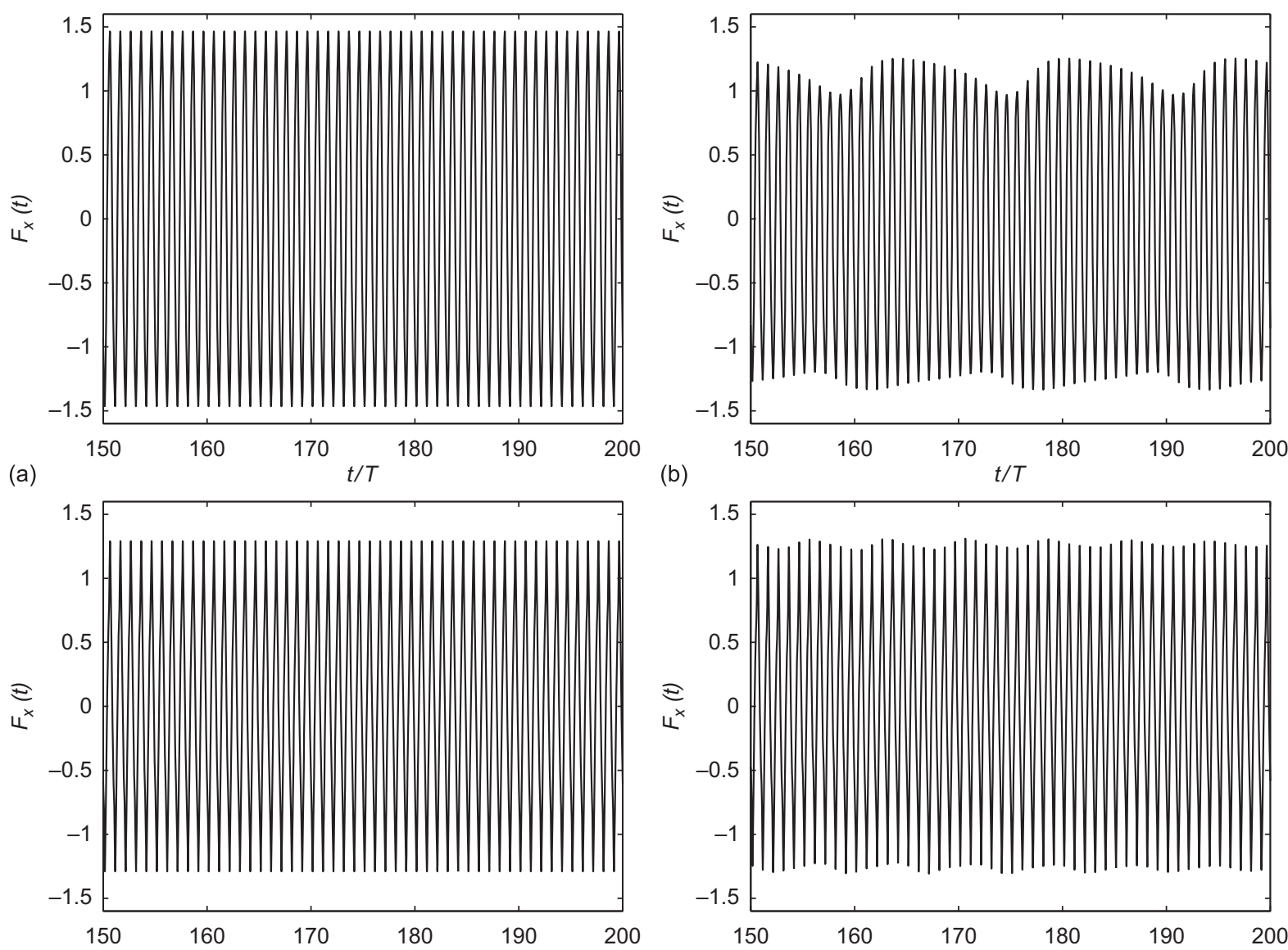

(b)

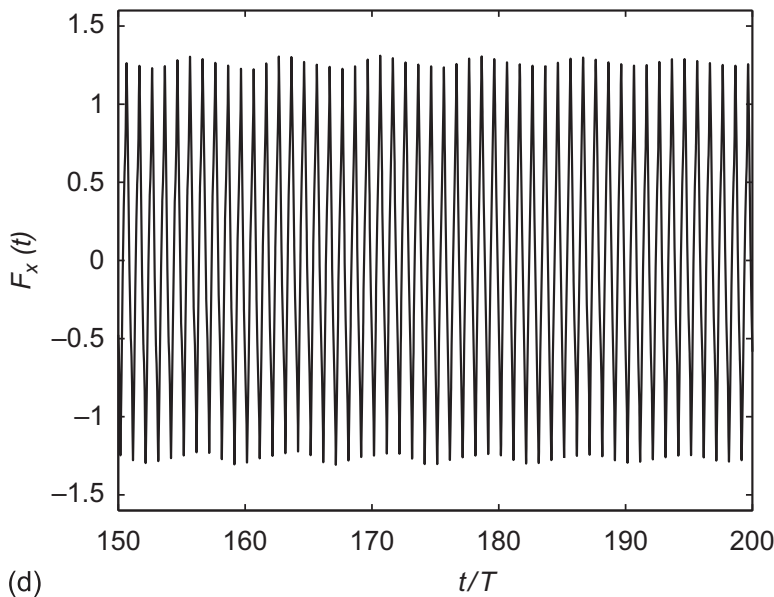

(c)

$t / T$

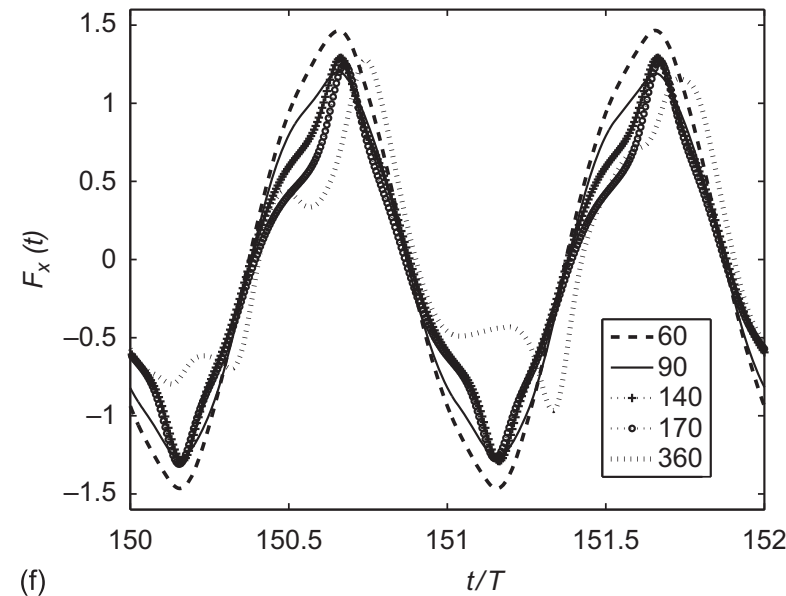

(e)

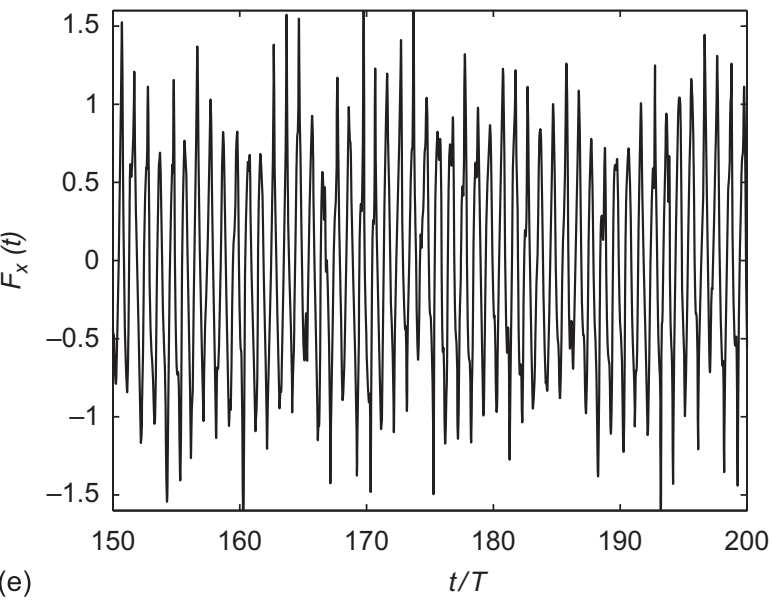

Fig. 3. (a)-(e) Time signals in steady state of the in-line force for $K C=10$ and different Re values between 40 and 500 representative of each regime. (f) Zoom of the five cases on two periods. (a) $R e=60$ representative of $40 \leqslant R e \leqslant 70$ (regime A), (b) $R e=90$ representative of $80 \leqslant R e \leqslant 100$ (regime $D$ ), (c) $\operatorname{Re}=140$ representative of $110 \leqslant \operatorname{Re} \leqslant 140$ (regime $F$ ), (d) $\operatorname{Re}=170$ representative of $150 \leqslant \operatorname{Re} \leqslant 280$ (regime $F$ ), (e) $\operatorname{Re}=360$ representative of $320 \leqslant \operatorname{Re} \leqslant 500$ (regime $G$ ) and (f) Zoom over two oscillation periods.

Firstly the spectral analysis confirms the existence of the dominant frequencies given by Williamson (1985). For $H_{x(f)}$ the main peak is always at the cylinder oscillation frequency $f_{0}=1 / T$. For $H_{y(f)}$ it occurs at frequency $2 f_{0}$ then $3 f_{0}$ in regime $\mathrm{D}$ then F. Furthermore, Figs. 6 and 7 reveal different spectral traductions of the force fluctuations observed in Figs. 3 and 4 . A significant feature of the range $80 \leq \operatorname{Re} \leq 100$ is the broadening of every harmonic of the frequency $f_{0}$ for both forces. For $\operatorname{Re} \geq 110$ even harmonics disappear and the remaining peaks at odd harmonics are again narrow. For Re $\geq 150$ (Figs. 6 (c) and $7(\mathrm{c})$ ), additional peaks appear at frequencies which are not multiples of the oscillation frequency. They correspond to 

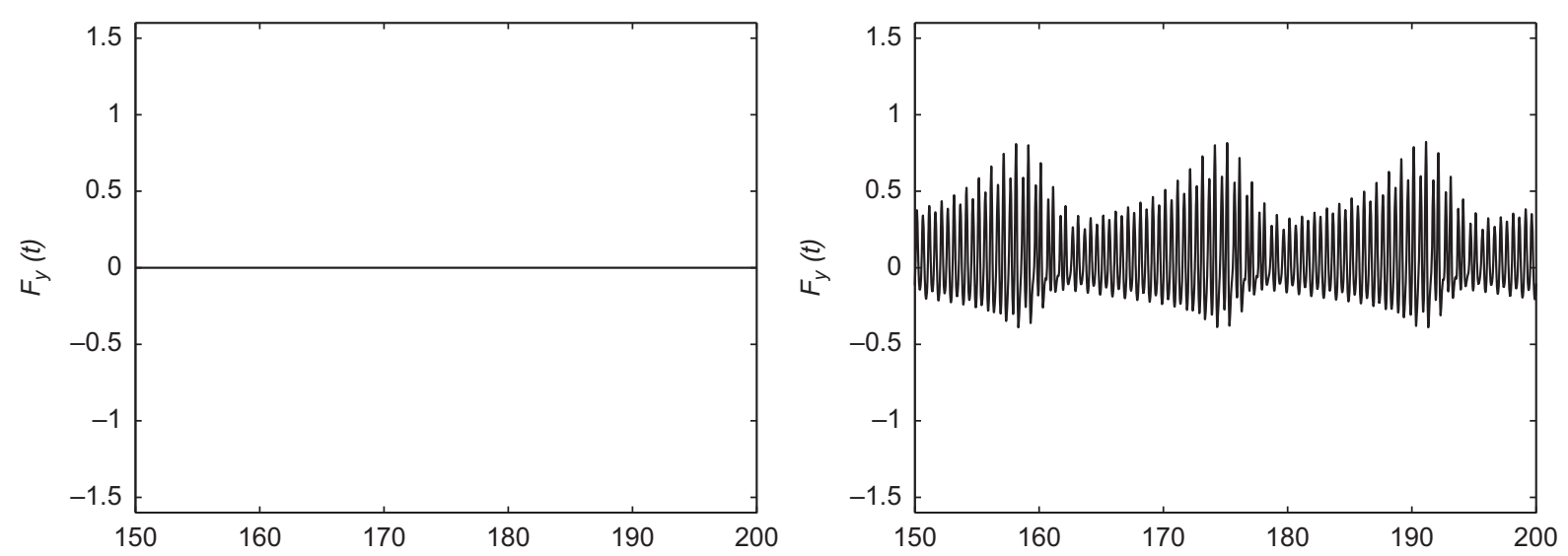

(a)

(b)
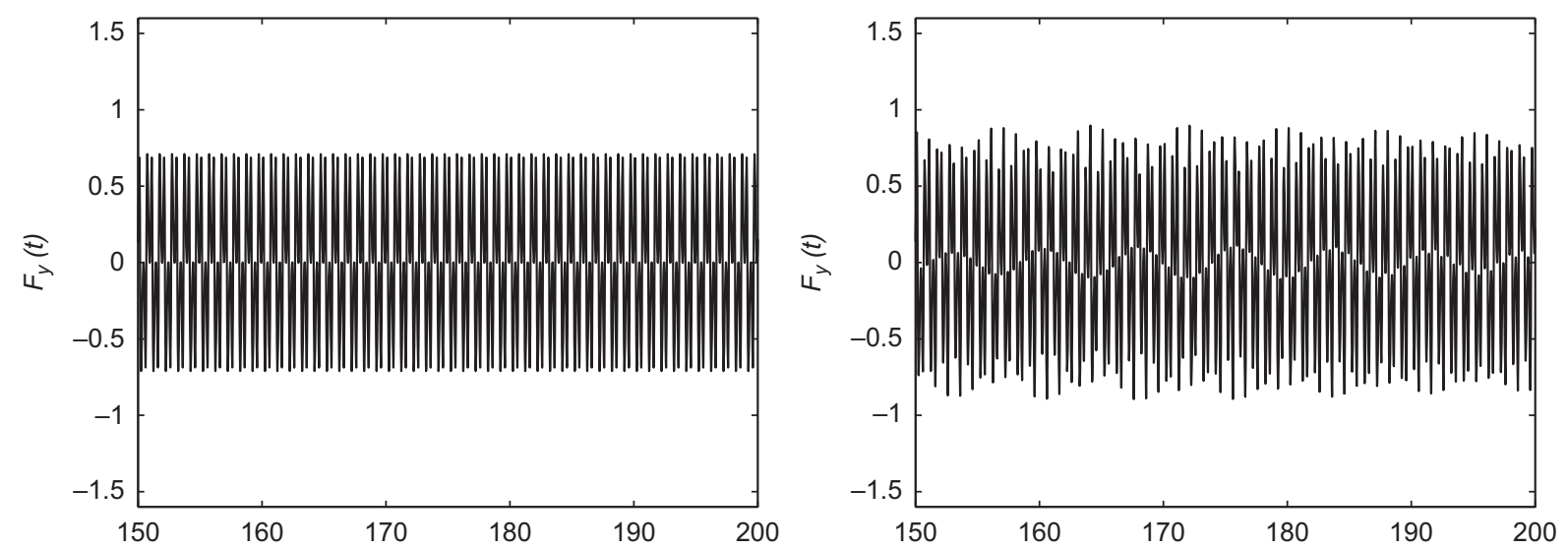

(c) $t / T$

(d)

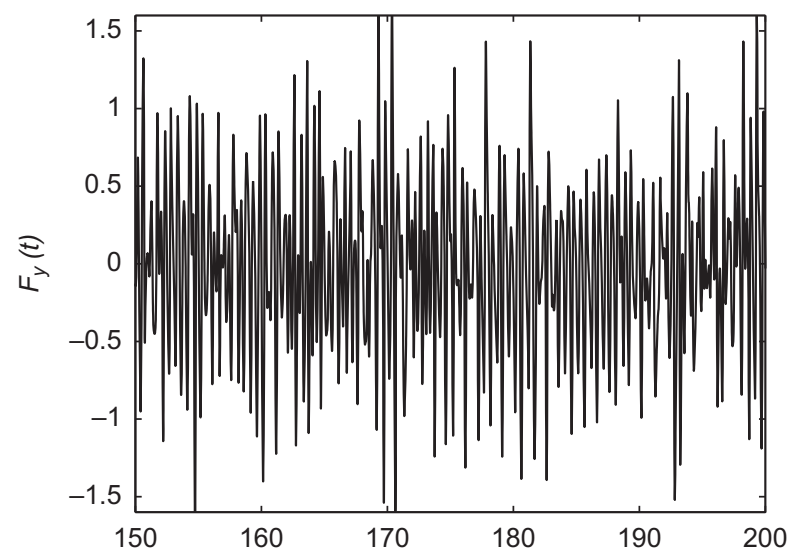

(e)

$t / T$

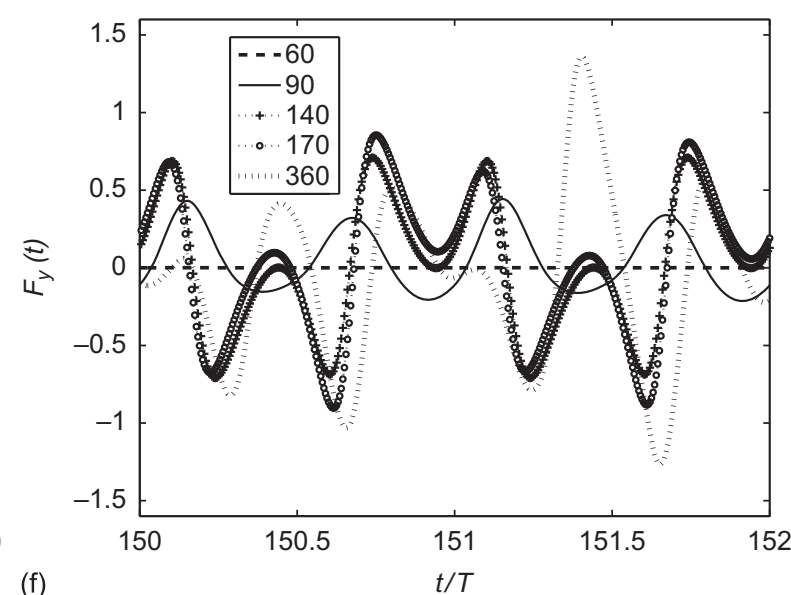

Fig. 4. (a)-(e) Time signals in steady state of the transverse force for $\mathrm{KC}=10$ and different Re values between 40 and 500 representative of each regime. (f) Zoom of the five cases on two periods. (a) $R e=60$ representative of $40 \leqslant \operatorname{Re} \leqslant 70$ (regime A), (b) $R e=90$ representative of $80 \leqslant R e \leqslant 100$ (regime D), (c) $R e=140$ representative of $110 \leqslant \operatorname{Re} \leqslant 140$ (regime F), (d) $R e=170$ representative of $150 \leqslant R e \leqslant 280$ (regime $F$ ), (e) $R e=360$ representative of $320 \leqslant R e-$ $\leqslant 500$ (regime $\mathrm{G}$ ) and (f) Zoom over two oscillation periods.

the force fluctuations in Figs. 3(d) and 4(d). Finally for Re $\geq 320$ the strong fluctuations observed in Figs. 3(e) and 4(e) yield quasi-continuous spectra in Figs. 6(d) and 7(d).

\subsubsection{Flow analysis}

In Sections 3.1.1 and 3.1.2, different force behaviors have been detected as Re increases. Here they are interpreted from flow dynamics using vorticity field (Fig. 8), as currently found in the literature. We suggest to distinguish the notions of 


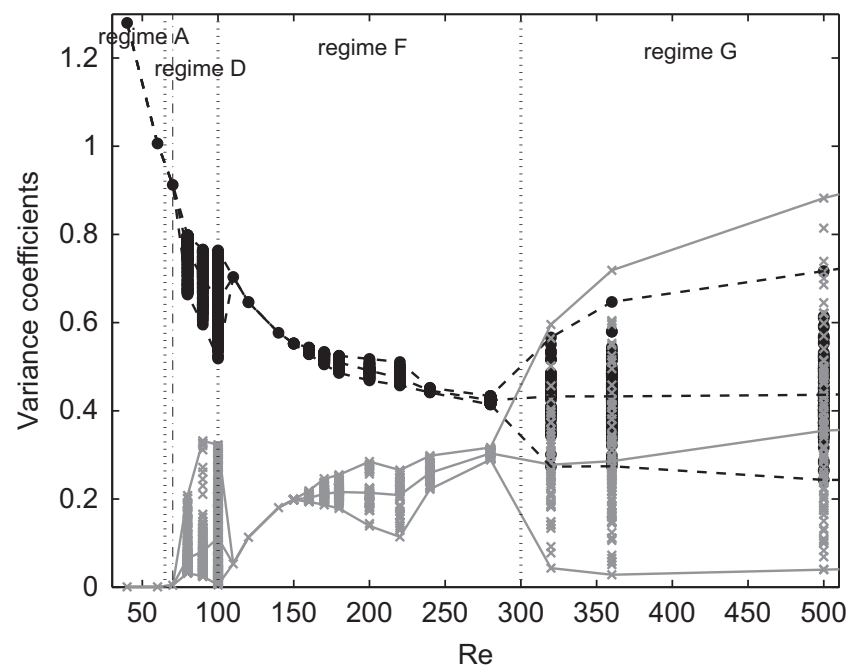

Fig. 5. Variance versus Re of the in-line and transverse forces coefficients: values for each $n$th computed period $\left\langle F_{x}(t)^{2}\right\rangle_{T_{n}}$ and $\left\langle F_{y}(t)^{2}\right\rangle_{T_{n}}($ circles: $x$-component; crosses: $y$-component), minimal, maximal and mean values (dashed line: $x$-component; solid line: $y$-component). Vertical lines indicate critical Re values between regimes supplied by Tatsuno and Bearman (1990, p. 161) (dotted) and Elston et al. (2006, p. 370) (dashed).
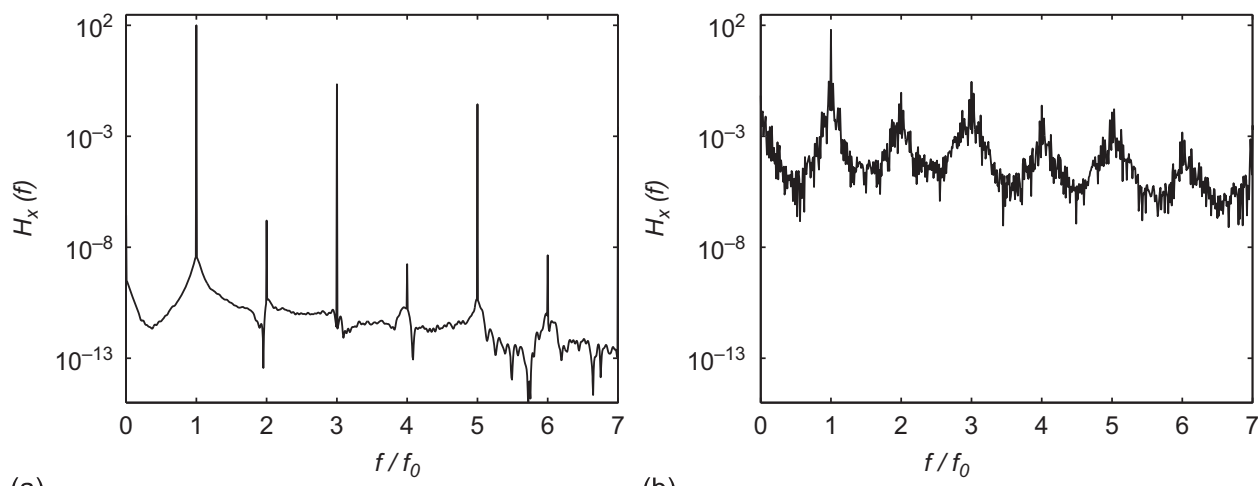

(a)

(b)
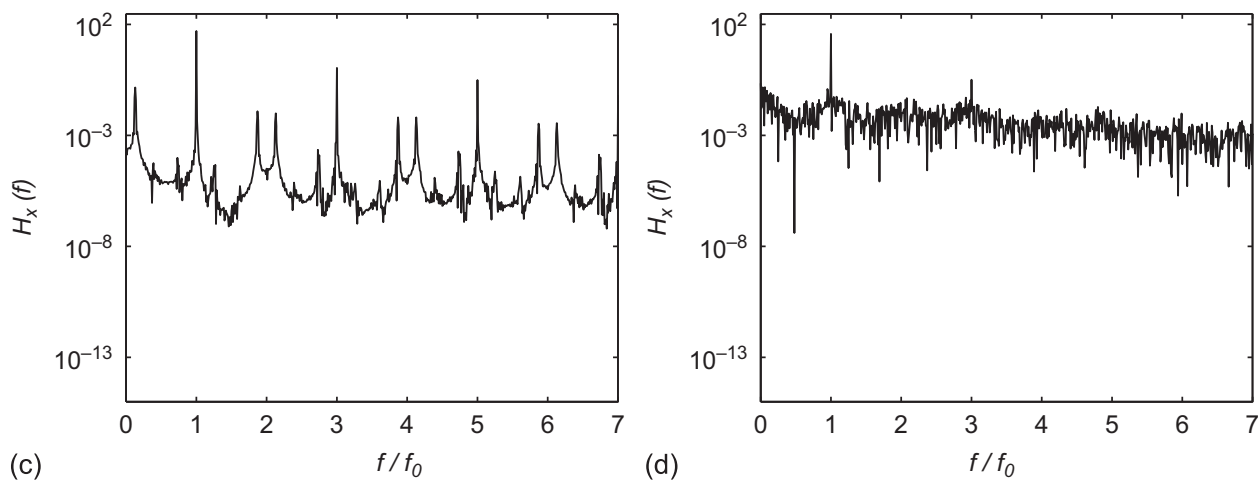

Fig. 6. Power spectral density of the in-line force $H_{x(f)}$ for $K C=10$ and different Re values between 40 and 500 representative of each regime.(a) Re=60 representative of $40 \leqslant \operatorname{Re} \leqslant 70$ (regime $A$ ), (b) $\operatorname{Re}=100$ representative of $80 \leqslant \operatorname{Re} \leqslant 100$ (regime D), (c) $\operatorname{Re}=170$ representative of $110 \leqslant \operatorname{Re} \leqslant 280$ (regime $\mathrm{F}$ ) and (d) $R e=360$ representative of $320 \leqslant R e \leqslant 500$ (regime $G$ ).

mode and regime. A mode refers to a specific flow pattern defined over a cylinder oscillation period. A regime is a system behavior observed over long durations in a certain region of the plane (KC, Re). This distinction introduces the question of the mode stability in the different regimes. In the range $40 \leq \mathrm{Re} \leq 500$ for $\mathrm{KC}=10$, four dominant modes are identified: symmetric, V-shaped, diagonal and chaotic. They appear in regimes A, D, F and G, respectively. In order to better analyze mode features and their links with force signals, we propose to use spatio-temporal diagrams of vorticity on the cylinder 

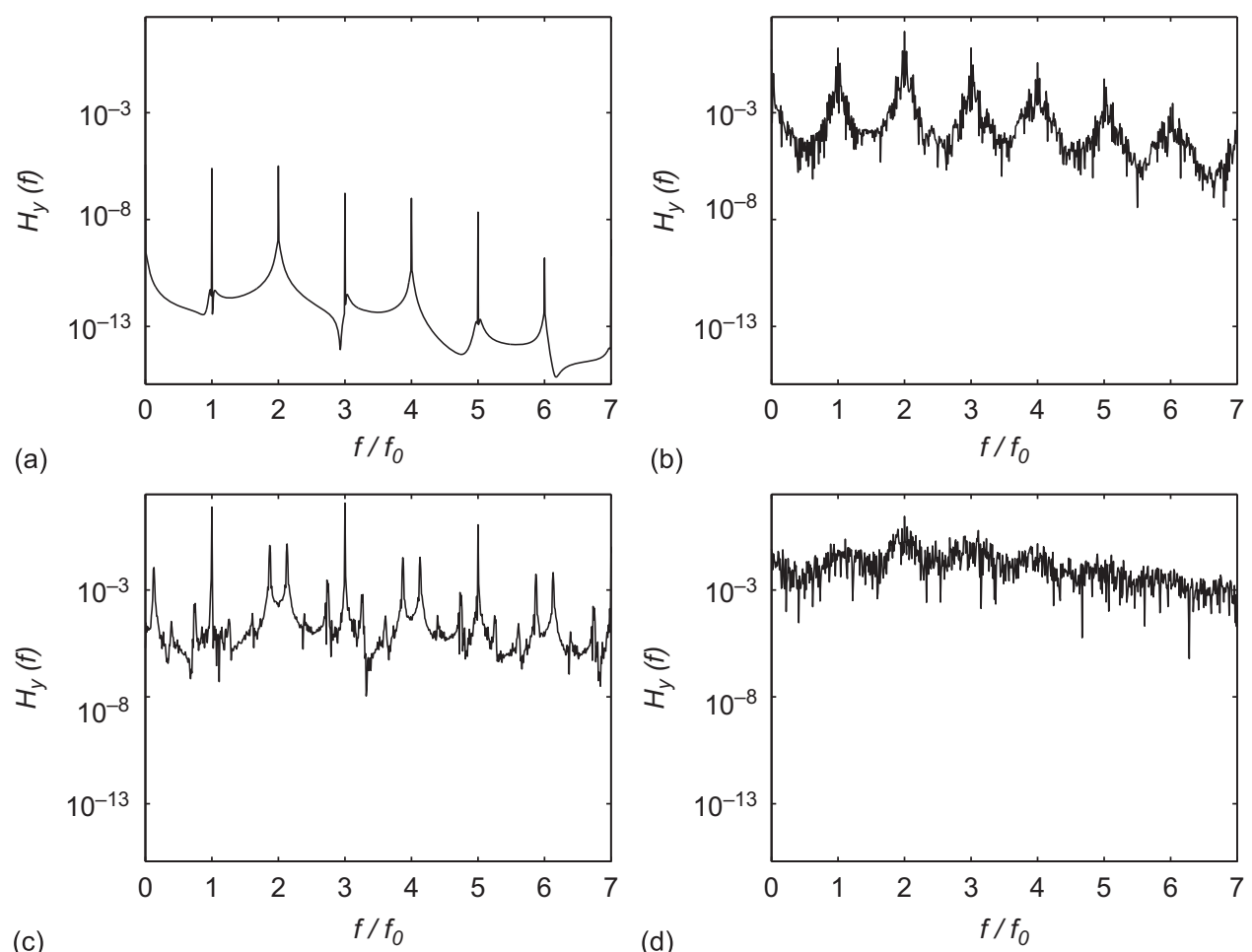

Fig. 7. Power spectral density of the transverse force $H_{y(f)}$ for $\mathrm{KC}=10$ and different Re values between 40 and 500 representative of each regime. (a) Re=60 representative of $40 \leqslant \operatorname{Re} \leqslant 70$ (regime $A$ ), (b) $\operatorname{Re}=100$ representative of $80 \leqslant \operatorname{Re} \leqslant 100$ (regime D), (c) $\operatorname{Re}=170$ representative of $110 \leqslant \operatorname{Re} \leqslant 280$ (regime $\mathrm{F}$ ) and (d) $\operatorname{Re}=360$ representative of $320 \leqslant \operatorname{Re} \leqslant 500$ (regime $G$ ).

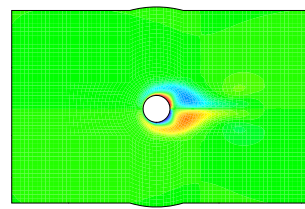

(a)

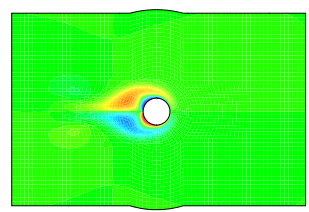

(b)

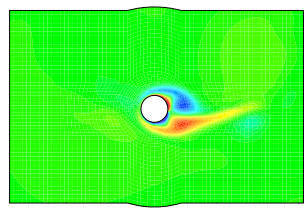

(c)

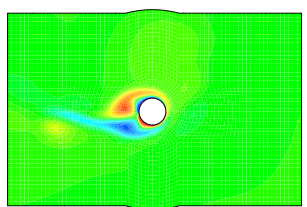

(d)

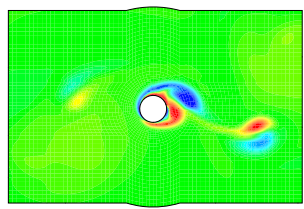

(e)

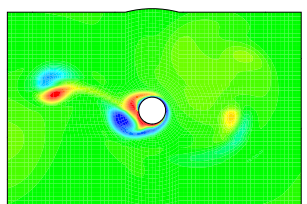

(f)

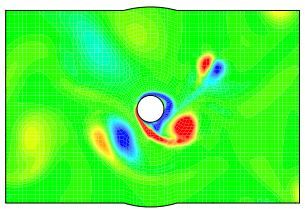

(g)

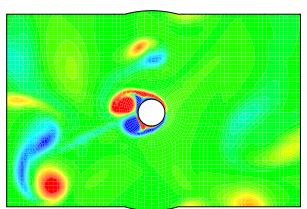

(h)

Fig. 8. Vorticity field around the cylinder at two consecutive half-cycles for the four dominant modes observed for KC $=10$ and $40 \leq$ Re $\leq 500$. The color bar is between -4 and +4 for every sub-figure. (a) $R e=60$ at $t=109.45 \mathrm{~T}$ representative of $40 \leqslant \operatorname{Re} \leqslant 70$ (regime $\mathrm{A}$ ), (b) $\mathrm{Re}=60$ at $\mathrm{t}=109.95 \mathrm{~T}$ representative of $40 \leqslant \operatorname{Re} \leqslant 70$ (regime $A$ ), (c) $R e=90$ at $t=149.45 \mathrm{~T}$ representative of $80 \leqslant R e \leqslant 100$ (regime $\mathrm{D}$ ), (c) $\operatorname{Re}=90$ at $\mathrm{t}=149.45 \mathrm{~T}$ representative of $80 \leqslant \mathrm{Re} \leqslant 100$ (regime D), e) $R e=170$ at $t=99.45 \mathrm{~T}$ representative of $110 \leqslant R e \leqslant 280$ (regime $F$ ), (f) $R e=170$ at $t=99.95 \mathrm{~T}$ representative of $110 \leqslant R e \leqslant 280$ (regime $F$ ), (g) $R e=360$ at $t=34.45 T$ representative of $320 \leqslant R e \leqslant 500$ (regime $G$ ) and $(h) R e=360$ at $t=34.95 T$ representative of $320 \leqslant R e \leqslant 500$ (regime $G$ ). (For interpretation of the references to color in this figure legend, the reader is referred to the web version of this article.)

surface (Fig. 9). The horizontal axis is the dimensionless time $t / T$. The vertical axis is the curvilinear abscissa $s$ on the cylinder defined by $s \in[0,1]$ clockwise, where $s=0$ at the point $(x=0, y=-D / 2)$ under the cylinder. Vorticity versus $t$ and $s$ is displayed with the same color bar for every sub-figure: anticlockwise (positive) vorticity is red, clockwise (negative) vorticity is blue, and null vorticity is green. It is nondimensionalized by dividing by $U_{0} / D$. The points of null vorticity are added in black. In Fig. 9, we focus on modes characterization, whereas their stability and force fluctuations on long characteristic times are specifically studied in Section 3.2.

Symmetric mode is encountered for $40 \leq \operatorname{Re} \leq 70$. Regarding the whole fluid domain (Figs. 8(a) and (b)), two weak symmetrical vortices are shed downstream the cylinder at each stroke. This is the pattern of regime A described by Tatsuno and Bearman (1990). The corresponding spatio-temporal diagram of vorticity on the cylinder (Fig. 9(a)) 

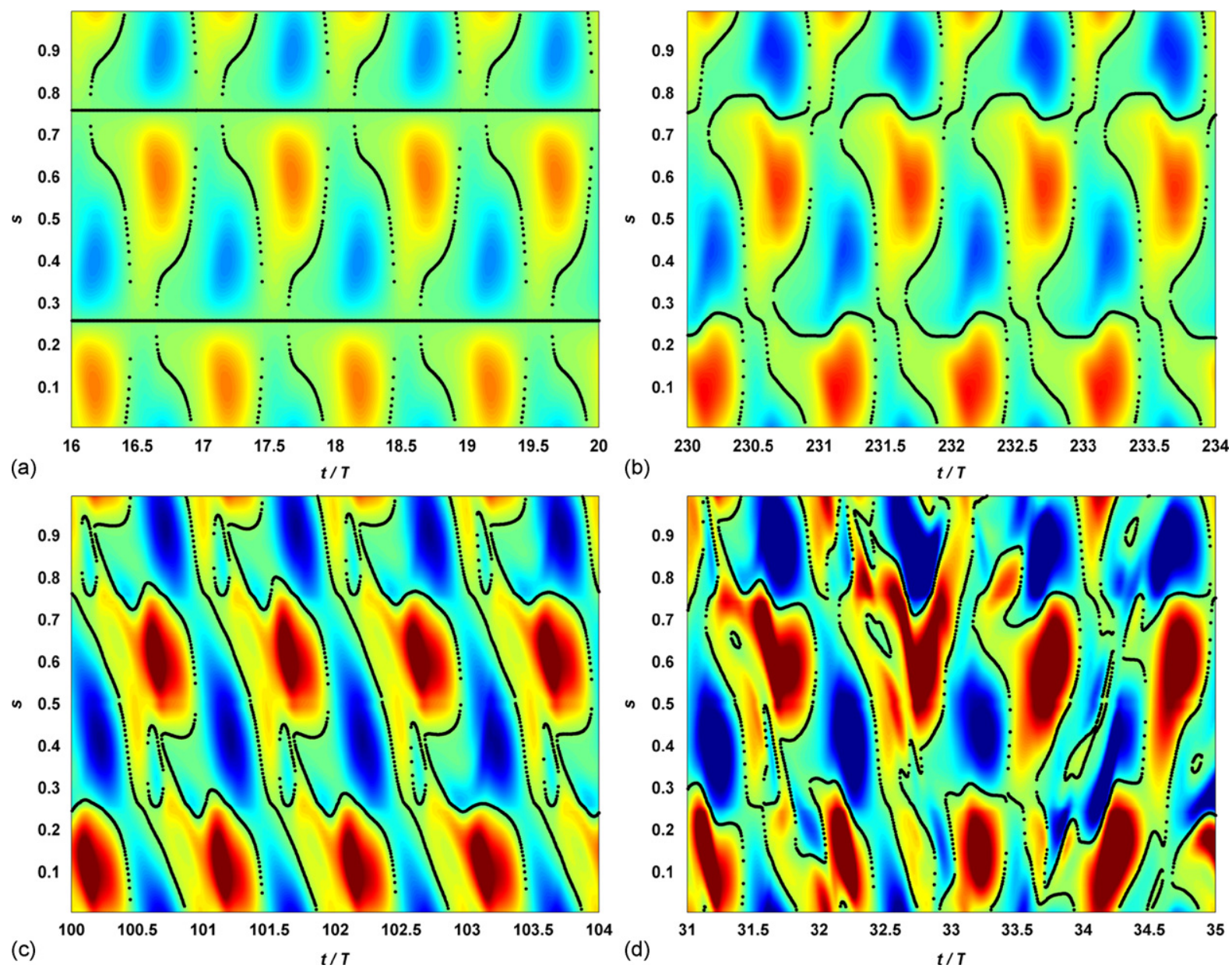

Fig. 9. Spatio-temporal diagrams of vorticity on the cylinder surface during four periods for the four dominant modes observed for KC $=10$ and $40 \leq \operatorname{Re} \leq 500$. The color bar is between -40 and +40 for every sub-figure. (a) $R e=40$ representative of $40 \leqslant \operatorname{Re} \leqslant 70$ (regime $A$ ), (b) $R e=80$ representative of $80 \leqslant \operatorname{Re} \leqslant 100$ (regime D), (c) $\operatorname{Re}=200$ representative of $110 \leqslant \operatorname{Re} \leqslant 280$ (regime $F$ ) and (d) $R e=360$ representative of $320 \leqslant \operatorname{Re} \leqslant 500$ (regime $G$ ). (For interpretation of the references to color in this figure legend, the reader is referred to the web version of this article.)

exhibits three kinds of symmetries (Elston et al., 2006). Firstly, we observe reflection symmetries about $s=0.25$ and 0.75 , (Eq. (6)) which corresponds to the symmetry about the axis of the cylinder oscillation $y=0$ in the global flow:

$$
\omega(0.25+s, t)=-\omega(0.25-s, t) \quad \text { and } \quad \omega(0.75+s, t)=-\omega(0.75-s, t),
$$

where $\omega$ is the $z$-component of the vorticity. Then we have a spatio-temporal symmetry corresponding to the reflection symmetry about the transverse axis $x=0$ with a phase shift of an half period

$$
\omega(1-s, t+0.5 T)=-\omega(s, t) .
$$

Thirdly, a spatio-temporal symmetry is associated to the central symmetry about the cylinder center with a phase shift of an half period

$$
\omega(s+0.5, t+0.5 T)=\omega(s, t) .
$$

Fig. 9(a) also presents two horizontal straight lines of points of null vorticity at $s=0.25$ and 0.75 . They are the front and rear stagnation points laying on the oscillation axis all cycle long. At each $t=0.15 T$ and $0.65 T$, two lines of zeros of vorticity appear from each side of the rear stagnation point, then diverge from it; it is the boundary layer separation then the growth of the recirculation zone. The two zeros of vorticity migrate over and under the cylinder until they reach the front stagnation point where they merge at each $t=0.45 T$ and $0.95 T$. Thus the lower and upper boundary layers are detached to constitute two vortices symmetrically shed from each side of the cylinder (Figs. 8(a) and (b)). Flow symmetries in Fig. 9(a) yield a large in-line force and a null transverse force as observed in Figs. 3(a) and 4(a). Indeed, according to Eq. (6), vorticity patterns are opposite under and over the oscillation axis. As a result their contributions cancel each other out for the transverse component and are added for the in-line component. 
The V-mode appears in Fig. 9(b) for the next range $80 \leq \mathrm{Re} \leq 100$ (regime D). In comparison with Fig. 9(a) presenting three symmetries given by Eqs. (6)-(8), only the relation Eq. (7) remains in Fig. 9(b). This symmetry is also present in global flow as shown in Figs. 8(c) and (d). Thus local symmetry properties on the cylinder surface are directly linked to symmetries of the global flow. They also enable to predict the parity of the transverse force. Indeed, according to Eq. (7), an element of vorticity in a given quadrant at $t$ reappears with opposite sign in the quadrant at the other side of the oscillation axis at $t+T / 2$. Thus its contribution to the transverse force is identical at $t$ and $t+T / 2$. As a result, time series of the transverse force presents four extrema per cycle (Fig. 4(f)) and the main peak of its spectrum is at frequency $2 f_{0}$ (Fig. $7($ b)). Besides vortex shedding can be identified from spatio-temporal diagram in Fig. 9(b) by merging of two lines of null vorticity. This is the detachment of a vorticity layer from the cylinder surface into a vortex. In Fig. 9(a), two symmetrical boundary layers were shed simultaneously at $t=0.45 T$ and $0.95 T$ for $s=0.25$ and 0.75 , respectively. Here in Fig. 9 (b), the merging of two lines of null vorticity occurs at $t=0.42 T$ and $0.92 T$ for $s=0.14$ and 0.86 . That produces the shedding of an anticlockwise (red) and a clockwise (blue) vortex, respectively, visualized in Figs. 8(c) and (d). They belong to the lower quadrants, like their shedding points $s=0.14$ and 0.86 .

A third mode, the diagonal mode (Figs. 8(e) and (f)), appears in the range $110 \leq \mathrm{Re} \leq 280$. It corresponds to regime D of Tatsuno and Bearman (1990) or double pair pattern of Williamson (1985). In the spatio-temporal diagram of vorticity (Fig. 9(c)) only the symmetry formulated by Eq. (8) is preserved. Like for the first two modes, symmetries are the same for the global flow and the cylinder surface. Besides, according to Eq. (8), an element of vorticity in a given quadrant at $t$ reappears with the same sign in the quadrant diametrically opposed at $t+T / 2$. Thus its contribution to the transverse force is opposite at $t$ and $t+T / 2$, yielding odd harmonics in the transverse force spectra (Fig. 7(c)). Again vortex shedding corresponds to the merging of two lines of null vorticity, at each $t=0.45 T$ and $0.95 T$, at $s=0.02$ and 0.52 , respectively. Those points are in opposite quadrants and define the direction of the diagonal pattern.

A last type of spatio-temporal diagram of vorticity occurs for Re $\geq 320$ (Fig. 9(d)). Every symmetry and periodicity property has disappeared. We call it the chaotic mode. Vorticity levels are high and reveal chaotic histories of vortices grown up from the boundary layers on the cylinder. In the same way, periodicity of flow dynamics in the whole domain disappears (Figs. 8(g) and (h)). Some patterns can be identified intermittently but they are not persistent. The lifetime of vortices is longer. They sometimes split and merge. Therefore interactions between vortices become more complex and unstable (Lam et al., 2010). The disordered spatio-temporal diagram of vorticity is also due to impacts of vortices on the cylinder, which interfere with the natural mechanism of vorticity creation.

\subsection{Fluctuations characterization}

Section 3.1 has pointed out that for some ranges of Re, forces fluctuate with characteristic times substantially larger than the cylinder oscillation period. To the best of our knowledge, that phenomenon has not been deeply investigated. It is studied here for regimes D, F and G successively.

\subsubsection{Fluctuations in the range $80 \leq \mathrm{Re} \leq 100$}

As mentioned in Section 3.1.3 the V-mode is dominant for $80 \leq \mathrm{Re} \leq 100$. But it intermittently appears the transverse and single-pair patterns observed by Williamson (1985), as more frequently as Re increases. Instead of being convected quasi-parallel to the oscillation axis as a V-pattern, vortices are shed with an angle of about $90^{\circ}$ (transverse street) or $45^{\circ}$ (oblique street).

The example of $\mathrm{KC}=10$ and $\mathrm{Re}=80$ is given in Figs. 10 and 11 . Vorticity isolines in the whole fluid domain show that the $\mathrm{V}$-mode is present from $t=60 T$ (Figs. 10(a) and (b)). It is progressively transformed into a transverse street from about $t=66 T$ (Figs. 10 (c) and (d)). At $t=70 T$, the transverse street is broken. During four cycles, vortices are shed in an oblique direction (Figs. 10(e) and (f)), then the V-mode reappears as in Figs. 10(a) and (b). Although those modes seem quite different regarding global flow, their signatures of vorticity on the cylinder are similar as exhibited by Fig. 11. It displays the spatio-temporal diagram of the points of null vorticity on the cylinder surface and the in-line and transverse forces for $t=60 T-78 T$. The different flow patterns in Fig. 10 are linked to oscillations of the stagnation points around $s=0.25$ and 0.75 (Fig. 11(a)). Their amplitude is correlated to the vortex shedding direction. Indeed, the same amount of vorticity is created at each cycle, but its spatial distribution varies. When the amplitude of the stagnation points increases, vortices are more compact and shed nearer from the bottom of the cylinder. Lateral vortex shedding of the V-shaped mode becomes transverse shedding, and vorticity is evacuated from the vicinity of the cylinder. The in-line force is then reduced and the transverse force increases (Fig. 11(b)) according to elementary hydrodynamics. It must be emphasized that characteristics of the spatio-temporal diagram of vorticity remain identical over the time. In particular the local symmetry relation Eq. (7) persists for the V-shaped, transverse and oblique modes. That means that they belong to the same spatio-temporal symmetry group.

\subsubsection{Fluctuations in the range $150 \leq \mathrm{Re} \leq 280$}

As shown in Figs. 3(d) and 4(d), amplitudes of the time signals of the forces fluctuate in the upper part of regime F for $\operatorname{Re} \geq 150$. This was not reported in the initial experiments of Tatsuno and Bearman (1990) but later indicated by some authors such as Dütsch et al. (1998), Uzunoğlu et al. (2001) and Elston et al. (2006). However, this was not analyzed more precisely. Amplitude fluctuations also appear in our simulations. Tests have been carried out for increasing sizes of the 


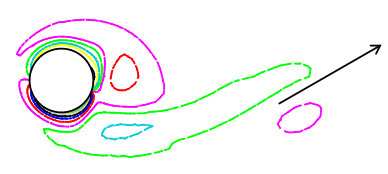

(a)

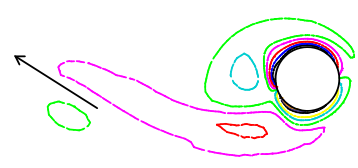

(b)

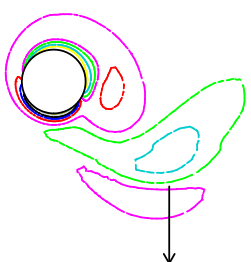

(c)

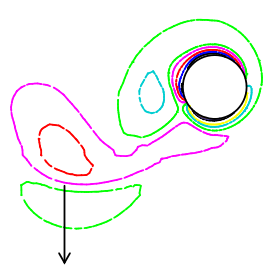

(d)

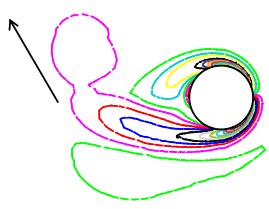

(e)

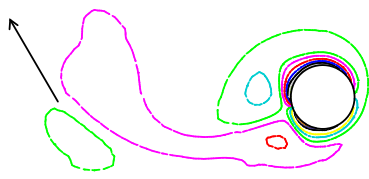

(f)

Fig. 10. Vorticity isolines around the cylinder for $K C=10$ and $R e=80$ at different instants illustrating the V-pattern (a and $b$ ), the transverse street (c and $d$ ) and the oblique street (e and f). Arrows indicate the directions in which vortices are convected. (a) $t=60.5 \mathrm{~T}$, (b) $t=61 \mathrm{~T}$, (c) $t=68.5 \mathrm{~T}$, (d) $t=69 \mathrm{~T}$, (e) $t=71.75 \mathrm{~T}$ and (f) $t=72 \mathrm{~T}$.

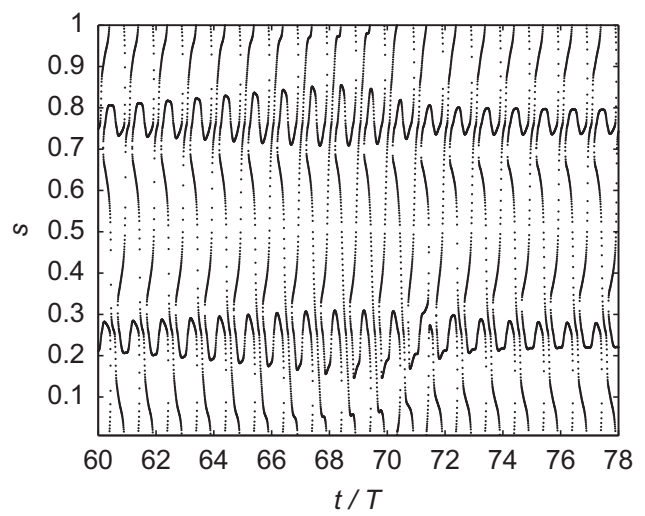

(a)

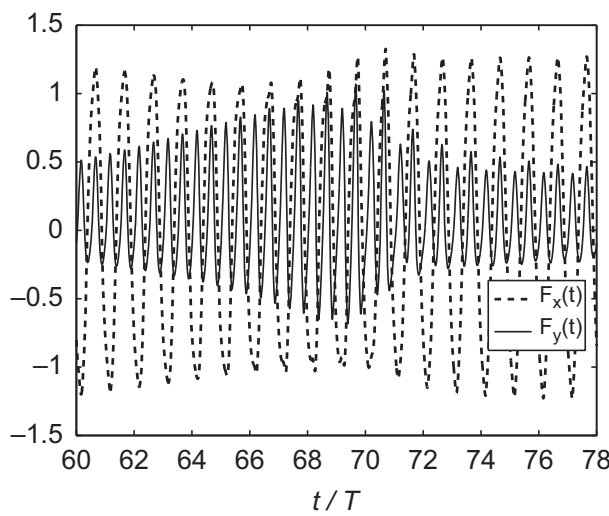

(b)

Fig. 11. (a) Spatio-temporal diagram of the points of null vorticity on the cylinder and (b) forces versus time for $K C=10$ and Re=80.

computational fluid domain. They have shown that fluctuations systematically occur, except if the computational domain is very small, which is not the case here. Therefore, fluctuations seem to result of a physical mechanism and not a numerical artifact.

Fig. 12 compares histories of the points of null vorticity on the cylinder surface with force signals. The case Re=180 is provided as example. Observation of the forces over a long duration in Fig. 12(b) lets appear amplitude fluctuations. From the flow point of view (Fig. 12(a)), force fluctuations are correlated to oscillations of the front and rear stagnation points around $s=0.25$ and 0.75 . Here for $\mathrm{Re}=180$, oscillation amplitudes of the stagnation point around $s=0.25$ (resp., 0.75 ) are the largest when oscillations of the point around $s=0.75$ (resp., 0.25 ) are the smallest. Then force fluctuations appear as the sum of a signal at low frequency (called carrier frequency) and a signal at the cylinder frequency. That will be referred to as a wavy fluctuation. For higher Re values the phase shift between the two stagnation points is reduced. Then force fluctuations appear as a modulation.

Now we quantitatively describe fluctuations using temporal and spectral signals of the in-line force. For Re $\geq 110$ we have seen in Section 3.1.2 that the main peaks of the in-line force spectrum were located at odd harmonics of the cylinder frequency $f_{0}=1 / T$. Such a spectrum can be fitted by the force expression proposed by Morison et al. (1950) (see Eq. (1)). For $\operatorname{Re} \geq 150$ (Fig. 6(c)) additional peaks appear at frequencies which are not multiples of the cylinder frequency. They are of the form $f_{c},(2 p) f_{0} \pm f_{c}$ and $(2 p+1) f_{0} \pm f_{m}$, where $f_{c}<1$ is the carrier frequency, $f_{m}<1$ the modulation frequency and $p \in \mathbb{N}^{*}$. Eq. (9) proposes an analytical expression of the in-line force to fit spectra obtained from simulations for $110 \leq \operatorname{Re} \leq 280$ :

$$
F_{x}^{\mathrm{fit}}(t)=\frac{\pi^{2}}{2 \mathrm{KC}} \alpha_{m} \cos \left(2 \pi f_{0} t\right)+\frac{1}{2}\left|\sin \left(2 \pi f_{0} t\right)\right|\left[\alpha_{d} \sin \left(2 \pi f_{0} t\right)+\beta_{c} \cos \left(2 \pi f_{c} t\right)+\beta_{m} \sin \left(2 \pi f_{0} t\right) \cos \left(2 \pi f_{m} t\right)\right],
$$

where the carrier coefficient $\beta_{c}$ and the modulation coefficient $\beta_{m}$ are determined empirically. Eq. (9) is based on the expression provided by Morison et al. (1950) (Eq. (1)) which is actually the first two terms. The third term is a wavy 


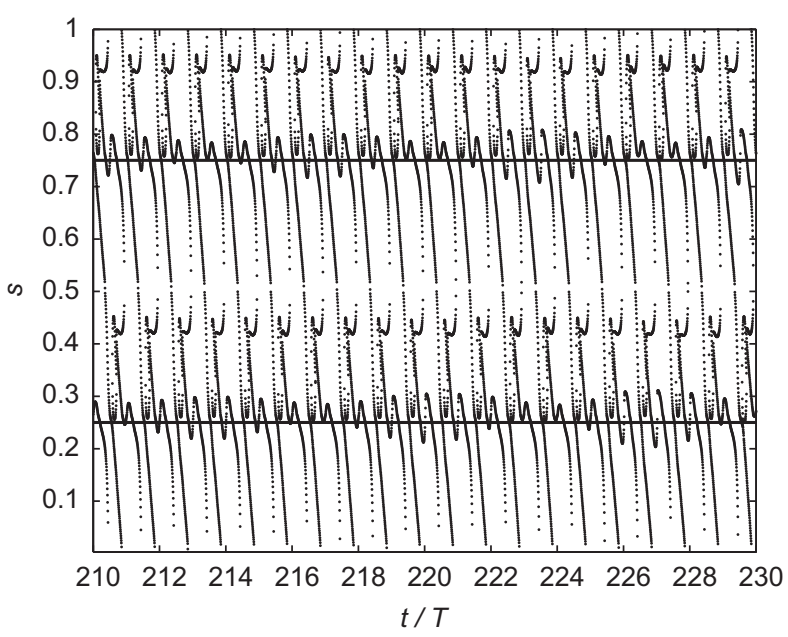

(a)

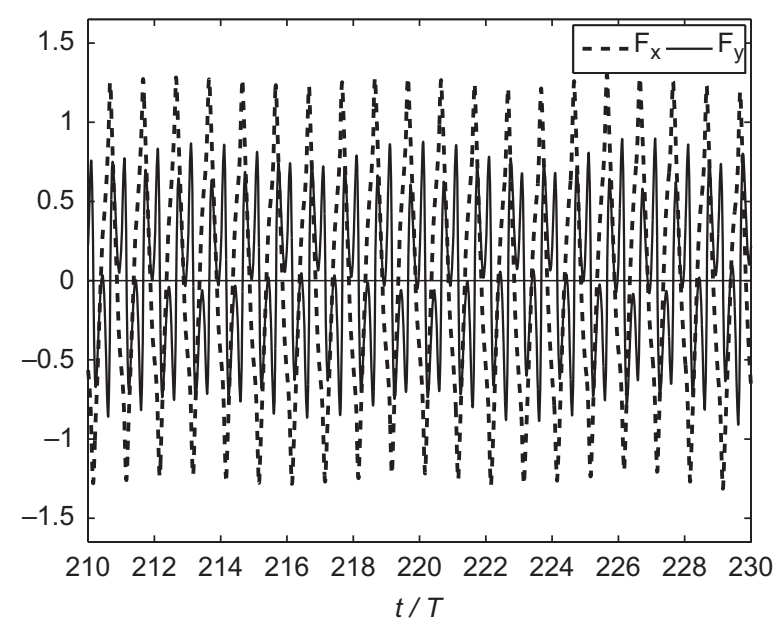

(b)

Fig. 12. Spatio-temporal diagram of the points of null vorticity on the cylinder (a) and forces versus time (b) for $\mathrm{KC}=10$ and $\mathrm{Re}=180$.

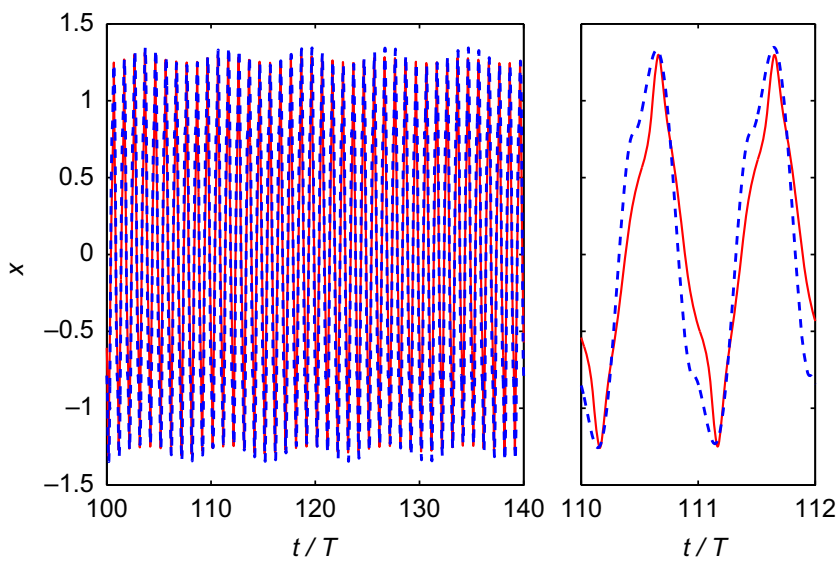

(a)

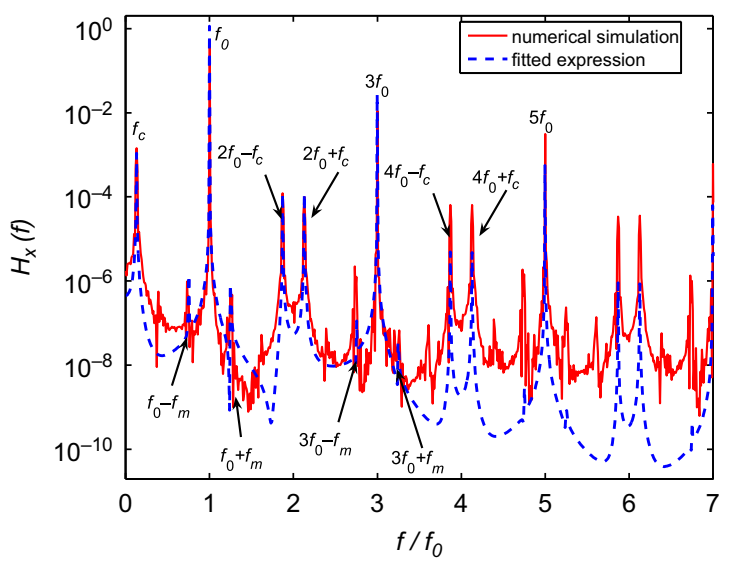

(b)

Fig. 13. (a) Evolution versus time and zoom over two periods, and (b) power spectral density versus frequency of the in-line force obtained by numerical simulation (solid line) and by Eq. (9) (dashed line) for $\mathrm{KC}=10$ and $\mathrm{Re}=170$.

fluctuation of the amplitude of the temporal signal at carrier frequency $f_{c}$. In spectral representation it appears as peaks at frequencies $f_{c}$ and $(2 p) f_{0} \pm f_{c}, p \in \mathbb{N}^{*}$. The last term of Eq. (9) describes an amplitude modulation of the temporal signal at frequency $f_{m}$, and peaks at frequencies $(2 p+1) f_{0} \pm f_{m}\left(p \in \mathbb{N}^{*}\right)$ in the force spectrum. An example illustrating the use of Eq. (9) is proposed in Fig. 13 for $\mathrm{Re}=170$. Parameters have been determined to fit temporal and spectral signals resulting of the numerical computation. Fig. 13 shows that main features of both temporal and spectral representations of the in-line force can be reproduced by Eq. (9).

The carrier and modulation coefficients $\beta_{c}$ and $\beta_{m}$ measured for each run are reported in Fig. 14(b) as a function of Re. For $\operatorname{Re}<150$, both coefficients are null since there is no fluctuations. For $150 \leq \operatorname{Re} \leq 200$, fluctuations appear rather as wavy fluctuations, because peaks around even harmonics $(2 p) f_{0} \pm f_{c}$ are higher than those around odd harmonics $(2 p+1) f_{0}$ $\pm f_{m}$. For $\operatorname{Re}>200$, the amplitude modulation becomes dominant over the wavy fluctuation. For Re $>280$ any phenomenon can be identified anymore because more and more peaks arise.

The carrier and modulation frequencies $f_{c}$ and $f_{m}$ are plotted versus Re in Fig. 14(b). The frequency resolution is $\Delta f=0.01 f_{0}$ according to Eq. (5). The carrier frequency $f_{c}$ increases with Re from 0.11 to $0.19 f_{0}$. A best fit of $f_{c}$ given by $f_{c} / f_{0}=f_{c 0}+a\left(\operatorname{Re}-\operatorname{Re}_{c}\right)$ yields $f_{c_{0}}=0.11, a=8.5 \times 10^{-4}$ and a critical Re number $\operatorname{Re}_{c}=150$. Since it depends on $\operatorname{Re}$, the carrier frequency seems to be linked to the natural response of the fluid and to the vortex formation time. On the contrary the modulation frequency $f_{m}$ is almost constant. We notice that it is a fraction $1 / 4$ of $f_{0}$. Then $f_{m}$ seems to be linked to the characteristic time of the structure. Appearance of coupling frequencies in force spectra was also observed by Morse and Williamson (2009) for the case of a cylinder controlled to oscillate transversely to a free stream. Although the configuration, and consequently flows and forces, are quite different from those considered here, the objectives and 


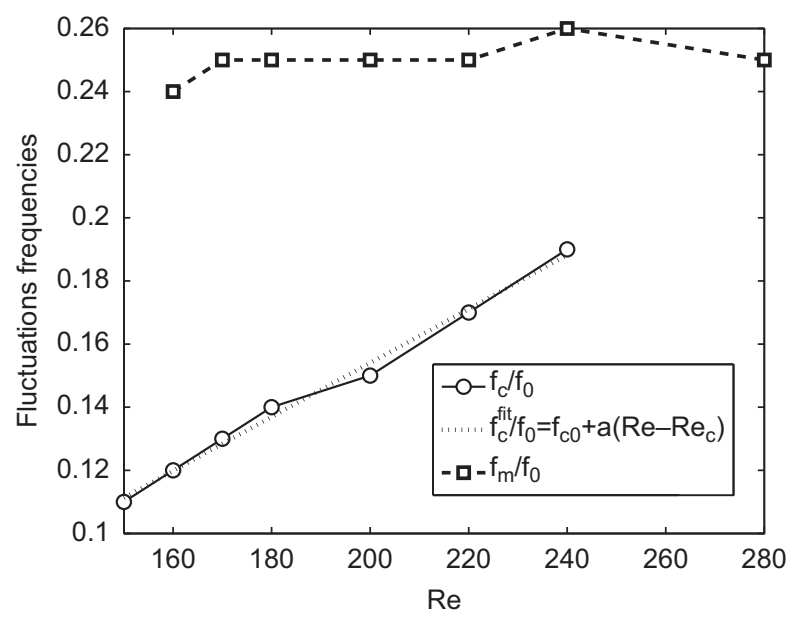

(a)

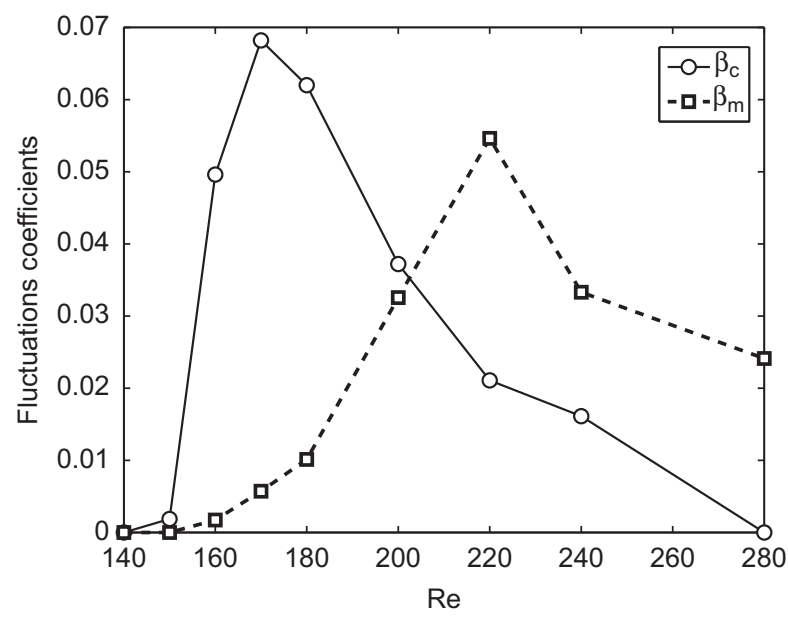

(b)

Fig. 14. (a) Carrier and modulation frequencies $f_{c}$ and $f_{m}$ and (b) carrier and modulation coefficients $\beta_{c}$ and $\beta_{m}$ versus Re from 150 to 280 .

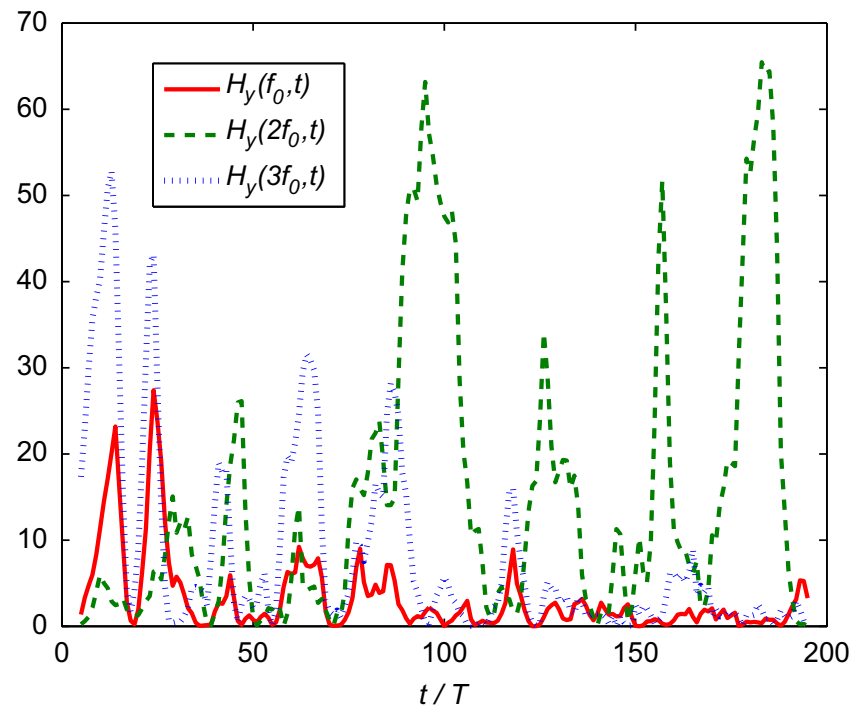

Fig. 15. Time evolutions of the amplitudes $H_{y}\left(f_{0}, t\right), H_{y}\left(2 f_{0}, t\right)$ and $H_{y}\left(3 f_{0}, t\right)$ of the power spectral density of the transverse force computed on running window of width $10 T$, at frequencies $f_{0}, 2 f_{0}$ and $3 f_{0}$, respectively, for $\mathrm{KC}=10$ and $\mathrm{Re}=360$.

approach of Morse and Williamson (2009) were similar to ours. Coupling frequencies were also interpreted as characteristic fluid times.

It must be precised that the transverse force also fluctuates in the same range $150 \leq \operatorname{Re} \leq 280$ as the in-line force (Fig. 4(d)). Peaks also appear in Fig. $7(\mathrm{c})$ at frequencies $f_{c},(2 p) f_{0} \pm f_{c}$ and $(2 p+1) f_{0} \pm f_{m}$ for $p \in \mathbb{N}^{*}$, where the carrier and modulation frequencies $f_{c}$ and $f_{m}$ take the same values as for the in-line force (Fig. 14(a)).

\subsubsection{Fluctuations in the range $320 \leq \mathrm{Re} \leq 500$}

According to Tatsuno and Bearman (1990) the range $320 \leq \operatorname{Re} \leq 500$ belongs to regime $\mathrm{G}$ (Fig. 1) characterized by a transverse street. However, as mentioned in Section 3.1.3, no persistent vortex pattern is observed here, producing a chaotic behavior. This is highlighted for the example of $\operatorname{Re}=360$ in Fig. 15 showing the amplitudes $H_{y}\left(f_{0}, t\right), H_{y}\left(2 f_{0}, t\right)$ and $H_{y}\left(3 f_{0}, t\right)$ of the power spectral density of the transverse force computed on running window of width $10 T$ at frequencies $f_{0}$, $2 f_{0}$ and $3 f_{0}$, where $H_{y}(f, t)$ is defined by

$$
H_{y}(f, t)=\left|\frac{1}{10 T} \int_{t-5 T}^{t+5 T} e^{-i 2 \pi f \tau} F_{y}(\tau) d \tau\right|^{2} .
$$


The dominant harmonic is $2 f_{0}$ or $3 f_{0}$ intermittently, revealing alternations between modes of the symmetry groups defined by Eqs. (7) and (8). Moreover, Iliadis and Anagnostopoulos (1998) also numerically studied three cases in regime G. They obtained different vortex patterns and concluded that the prominent characteristic of that regime was the switching of modes at different cycles. The results of Iliadis and Anagnostopoulos (1998) and those of the present study lead to think that regime $G$ cannot be reduced to the transverse vortex street pattern as proposed first by Tatsuno and Bearman (1990), but should be defined as an aperiodic regime with unstable vortex patterns.

\section{Discussion}

Low frequency amplitude fluctuations of the force exerted on the cylinder occur for $80 \leq \operatorname{Re} \leq 100$ and $150 \leq \operatorname{Re} \leq 280$. The fluid force is a global quantity which reflects global flow dynamics, as can be seen in Figs. 11 and 12, where vorticity dynamics is correlated to the force. The appearance of amplitude fluctuations is related to some global flow instabilities. This provides new features to regime $\mathrm{D}$ whose V-pattern becomes the most persistent but not the only one observed, and splits regime $\mathrm{F}$ into two sub-regimes, a first synchronous and a second with low frequency fluctuations. We propose to discuss physical meanings of those fluctuations.

Each flow regime can be characterized by spatio-temporal symmetry relations associated to one or several modes. Regime $\mathrm{A}$ is the existence domain of the symmetric mode. It is stable and forces are periodic. At $\mathrm{Re}=70$ flow bifurcates to another periodic mode but with a two-dimensional symmetry breaking as expected by the two-dimensional Floquet stability analysis conducted by Elston et al. (2004). As Re increases in regime D, force spectra exhibit a spectral broadening (Figs. 6(b) and 7(b)) leading to a very low frequency modulation of the two-dimensional flow. This modulation instability is due to the permutation of three modes (V-shaped, transverse and oblique, see Fig. 10). It is worth noticing that those three modes belong to the symmetry group given by Eq. (7) which is a key feature of regime D. The history of vorticity production on the cylinder surface shows that the regions that are sensitive to the modulation are the positions of both the rear and front stagnation points. Our study only deals with two-dimensional flows while Elston et al. (2006) showed that regime $\mathrm{D}$ was unstable to three-dimensional perturbations. Then one may ask whether the modulation instability observed in the two-dimensional flow is relevant in the case of real three-dimensional flows. Nehari et al. (2004) reported in a three-dimensional simulation the existence of mode switching to explain force fluctuations they observed in regime D, which is consistent with our results. It is then possible that this modulation instability as described here be a new ingredient that could be considered for further stability analyses of the three-dimensional flow.

Regime $\mathrm{F}$ is the existence domain of symmetry Eq. (8), which only comprises the diagonal mode. It is observed in the global flow field at every investigated Re value of regime $\mathrm{F}$ and at every time. The transition from regime $\mathrm{D}$ to $\mathrm{F}$ corresponds to a two-dimensional symmetry breaking, synchronous with the cylinder frequency. As Re increases, force fluctuations appear for Re $\geq 150$ in the middle and upper part of regime F, as shown by the comparison of Figs. 3(c) and (d), or 4 (c) and (d). In spectral representation this is related to the appearance of two distinct low frequencies that non-linearly interact with harmonics of the cylinder frequency. This global flow instability is not easily detectable from wake patterns since only the diagonal mode is observed in regime F. We checked that the low frequencies are also present in velocity measurements at points distant of few diameters from the cylinder. This flow instability is also intimately related to the oscillations of the stagnation points on the cylinder surface (Fig. 12). Dütsch et al. (1998) and Nehari et al. (2004) also reported low frequency fluctuations for three-dimensional flows in regime $\mathrm{F}$. Once again the transition at $\operatorname{Re}=150$ observed for two-dimensional flows may be relevant in the case of three-dimensional flows.

\section{Conclusion}

A finite element method was applied to solve the two-dimensional Navier-Stokes equations in the case of a circular cylinder oscillating in a fluid initially at rest. The objective was to investigate the influence of increasing Re from 40 to 500 at $K C=10$. Five ranges of Re appear from temporal and spectral analyses of the in-line and transverse forces. Transitions between regimes mainly consist in changes between periodic and aperiodic behaviors, modifications of the distribution of energy in force spectra, and changes in spatio-temporal symmetries. Two regimes are also characterized by amplitude fluctuations of the forces with long typical times. Those phenomena had been indicated but not specifically studied in the literature. Fluctuations appear here as instabilities of flow modes associated to symmetry relations. For future investigations, computations for other $\mathrm{KC}$ values could be carried out in order to determine the extension of the domains $(\mathrm{KC}, \mathrm{Re})$ where amplitude fluctuations of the forces appear. The present study is to be continued with three-dimensional computations in order to determine if the present instabilities are still observed, or if they are the two-dimensional equivalent of a three-dimensional instability. The transitions between regimes $\mathrm{D}, \mathrm{F}$ and $\mathrm{G}$ could also be more precisely investigated.

\section{Acknowledgments}

This work was supported by DISN/R4G at CEA through the program RNRNa. The authors thank Emmanuel de Langre for his stimulating and thoughtful discussion. 


\section{References}

Anagnostopoulos, P., Minear, R., 2004. Blockage effect of oscillatory flow past a fixed cylinder. Applied Ocean Research $26,147-153$.

Dütsch, H., Durst, F., Becker, S., Lienhart, H., 1998. Low-Reynolds-number flow around an oscillating circular cylinder at low Keulegan-Carpenter numbers. Journal of Fluid Mechanics 360, 249-271.

Elston, J., Blackburn, H., Sheridan, J., 2006. The primary and secondary instabilities of flow generated by an oscillating circular cylinder. Journal of Fluid Mechanics 550, 359-389.

Elston, J., Sheridan, J., Blackburn, H., 2004. Two-dimensional Floquet stability analysis of the flow produced by an oscillating circular cylinder in quiescent fluid. European Journal of Mechanics B/Fluids 23, 99-106.

Guilmineau, E., Queutey, P., 2002. A numerical simulation of vortex shedding from an oscillating circular cylinder. Journal of Fluids and Structures 16 $773-794$.

Iliadis, G., Anagnostopoulos, P., 1998. Viscous oscillatory flow around a circular cylinder at low Keulegan-Carpenter numbers and frequency parameters. International Journal for Numerical Methods in Fluids 26, 403-442.

Justesen, P., 1991. A numerical study of oscillating flow around a circular cylinder. Journal of Fluid Mechanics 222, $157-196$.

Lam, K., Dai, G., 2002. Formation of vortex street and vortex pair from a circular cylinder oscillating in water. Experimental Thermal and Fluid Science 26 , 901-915.

Lam, K., Hu, J., Liu, P., 2010. Vortex formation processes from an oscillating circular cylinder at high Keulegan-Carpenter numbers. Physics of Fluids 22.

Lin, X., Bearman, P., Graham, J., 1996. A numerical study of oscillatory flow about a circular cylinder for low values of beta parameter. Journal of Fluids and Structures 10, 501-526.

Morison, J., O’Brien, M., Johnson, J., Schaaf, S., 1950. The forces exerted by surface waves on piles. Petroleum Transactions, AIME 189, 149-157.

Morse, T., Williamson, C., 2009. Fluid forcing, wake modes, and transitions for a cylinder undergoing controlled oscillations. Journal of Fluids and Structures 25, 697-712.

Nehari, D., Armenio, V., Ballio, F., 2004. Three-dimensional analysis of the unidirectional oscillatory flow around a circular cylinder at low KeuleganCarpenter and beta numbers. Journal of Fluid Mechanics 520, 157-186.

Obasaju, E., Bearman, P., Graham, J., 1988. A study of forces, circulation and vortex patterns around a circular cylinder in oscillating flow. Journal of Fluid Mechanics 196, 467-494.

Sarpkaya, T., 2005. On the parameter $\beta=\operatorname{Re} / K C=D^{2} / v T$. Journal of Fluids and Structures 21, 435-440.

Smith, P., Stansby, P., 1991. Viscous oscillatory flows around cylindrical bodies at low Keulegan-Carpenter numbers using the vortex method. Journal of Fluids and Structures 5, 339-361.

Sun, X., Dalton, C., 1996. Application of the LES method to the oscillating flow past a circular cylinder. Journal of Fluids and Structures 10 , 851-872.

Tatsuno, M., Bearman, P., 1990. A visual study of the flow around an oscillating circular cylinder at low Keulegan-Carpenter numbers and low Stokes numbers. Journal of Fluid Mechanics 211, 157-182.

Uzunoǧlu, B., Tan, M., Price, W., 2001. Low-Reynolds-number flow around an oscillating circular cylinder using a cell viscous boundary element method. International Journal for Numerical Methods in Engineering 50, 2317-2338.

Vuik, C., Segal, A., 2006. Finite element methods for the incompressible Navier-Stokes equations. Technical Report, Delft Institute of Applied Mathematics.

Wang, C., 1968. On high frequency oscillatory viscous flows. Journal of Fluid Mechanics 32, 5-68.

Williamson, C., 1985. Sinusoidal flow relative to circular cylinders. Journal of Fluid Mechanics 155, 141-174

Zhang, H., Zhang, X., 1997. Flow structure analysis around an oscillating circular cylinder at low KC number: a numerical study. Computers and Fluids 26, 83-106 